\title{
ON THE DEPTH OF LOCAL RINGS OF INVARIANTS OF CYCLIC GROUPS
}

\author{
JOHN FOGARTY
}

\begin{abstract}
For wild actions of a cyclic group $G$ on a local ring $R$, with $R^{G}$ noetherian, it is shown that depth $R-\operatorname{depth} R^{G}$ can be arbitrarily large, even if $R$ is regular and contains $\underline{Z}$.
\end{abstract}

1. Introduction. Let $R$ be a noetherian local ring and let $G$ be a finite group of automorphisms of $R$. If the ring $R^{G}$ of $G$-invariants in $R$ is noetherian, what is the relation between the depth of $R$ and the depth of $R^{G}$ ? (For the problem of when $R^{G}$ is noetherian, see [1].) This question has been raised in connection with problems of unique factorization (see [4, esp. \$4]). The theme of this note is that when the residue field characteristic of $R$ divides the order of $G$, things are as bad as possible, at least if one expects that the depth of $R$ will govern the depth of $R^{G}$. We exhibit a class of actions of a cyclic group $G$ of order $p^{n}$ in characteristic $p$ for which the depth of $R^{G}$ is 2 , provided the depth of $R$ is at least 2 . There is also a related phenomenon in mixed characteristic where the ring of invariants always has depth $\leqslant 3$.

2. The depth of $R^{G}$. Recall that if $R$ is a noetherian local ring with maximal ideal $\mathrm{m}$, the depth of $R$ is the largest integer $d \geqslant 0$ for which there exists a sequence $f_{0}$, $f_{1}, \ldots, f_{d}$ of elements of $m$ with $f_{0}=0$ and $\left(\left(f_{0}, \ldots, f_{i-1}\right):\left(f_{i}\right)\right)=\left(f_{0}, \ldots, f_{i-1}\right)$, $1 \leqslant i \leqslant d$.

Let $G$ be a finite group of automorphisms of $R$ such that $R^{G}$ is noetherian. Let $g$ be the order of $G$.

Proposition 1. If $g$ is invertible in $R$, then $\operatorname{depth}\left(R^{G}\right)>\operatorname{depth}(R)$.

PRoof. If $\operatorname{depth}(R)=0$, there is nothing to prove, so assume that $\operatorname{depth}(R)>0$ and let $f \in \mathfrak{m}$ be a non-zero-divisor. Then $F=\operatorname{Norm}(f)$ is a non-zero-divisor in $R^{G}$ and $(F R)^{G}=F R^{G}$. Now $\operatorname{depth}(R / F R)=\operatorname{depth}(R)-1$ and $\operatorname{depth}\left(R G / F R^{G}\right)$ $=\operatorname{depth}\left(R^{G}\right)-1$. Since $g$ is invertible in $R,(R / F R)^{G}=R^{G} / F R^{G}$ and the proposition follows by induction on the depth.

That the inequality in Proposition 1 may be strict is illustrated by the following simple example. Let $k$ be the complex field and let $R=k[[X, Y]] /\left(X Y, Y^{2}\right)$. Let $G$ be the group generated by the automorphism, $X \mapsto X, Y \mapsto-Y$, of $R$. $R$ has depth 0 and $R^{G}=k[[X]]$ has depth 1 . 
Since $R$ is integral over $R^{G}$, one always has $\operatorname{dim}\left(R^{G}\right)=\operatorname{dim}(R)$, so that if $g$ is invertible in $R$, then $R$ Cohen-Macaulay $\Rightarrow R^{G}$ Cohen-Macaulay. In the case where $g$ may not be invertible in $R$, all that can be said in general is

Proposition 2. Let $d$ be one of 0,1 or 2. If $\operatorname{depth}(R)>d$, then $\operatorname{depth}\left(R^{G}\right)>d$.

Proof. The case $d=0$ is obvious. If $d=1$, let $f$ be a non-zero-divisor in the maximal ideal of $R$. Then, as before, $F=\operatorname{Norm}(f)$ is a non-zero-divisor in $R^{G}$ and $(F R)^{G}=F R^{G}$. If $d=2$, then $\operatorname{depth}(R / F R) \geqslant 1$. If $h$ is a non-zero-divisor in the maximal ideal of $(R / F R)^{G}$, then $h^{g}$ is a non-zero-divisor in $R^{G} / F R^{G}$. Hence there is an $H$ in the maximal ideal of $R^{G}$ such that $F, H$ is an $R^{G}$-sequence.

We show below that for every $d \geqslant 2$, there is an $R$ of depth $d$ with $R^{G}$ of depth 2. For this, we need the following version of the normal basis theorem.

LEMMA 3. Let $S$ be a noetherian semilocal domain and let $G$ be a finite group of automorphisms of $S$ operating transitively on the maximal ideals of $S$. If $S$ is a finite étale extension of $R=S^{G}$ then there exists an $f \in S$ such that $\left\{f^{\circ}: \sigma \in G\right\}$ is a free basis of $S$ over $R$.

Proof. Since $G$ acts transitively on the maximal ideals of $S$ and $S$ is faithfully flat over $R$, it follows that $R$ is a noetherian local ring. Let $m$ be its maximal ideal. Then $S / \mathfrak{m} S$ is a direct sum of finite separable extensions $K_{i}=S / \mathfrak{n}_{i}$ of $R / \mathfrak{m}=k$, $\mathfrak{n}_{1}, \ldots, \mathfrak{n}_{r}$ being the maximal ideals of $S$. Set $G_{j}=\left\{\sigma \in G: \sigma \equiv 1 \bmod \mathrm{m}^{j} S\right\}$. Then $G_{n}=(1)$ for large $n$. Fix such an $n$. If $G_{1}$ contains a subgroup $H$ whose order $k$ is invertible in $S / \mathfrak{m} S$, then since $\left(S / \mathrm{m}^{n} S\right)^{H}$ maps onto $S / \mathrm{m} S$, and $S / \mathrm{m}^{n} S$ is a finite $R / \mathfrak{m}^{n}$-module, it follows from Nakayama's lemma that $\left(S / \mathfrak{m}^{n} S\right)^{H}=S / \mathfrak{m}^{n} S$, so that $H=(1)$. Thus if $k$ has characteristic 0 , then $G_{1}=(1)$ and if $k$ has characteristic $p>0$, then $G_{1}$ is a $p$-group. In the latter case $G_{1}$ is still trivial. To see this, note that $G_{1}$ permutes transitively the maximal ideals of $S$ over a given maximal ideal of $S^{G_{1}}$, for if $\mathfrak{n}_{i}^{G_{1}}=\mathfrak{n}_{j}^{G_{1}}$ and $f \in \mathfrak{n}_{i}$ then $\Pi_{\sigma \in G_{1}} f^{o} \in \mathfrak{n}_{j}$ so $f^{t} \in \mathfrak{n}_{j}$ for some $t \in G_{1}$, i.e., $\mathfrak{n}_{i} \subset \cup_{\sigma \in G_{1}} \mathfrak{n}_{j}^{\sigma}$, and so $\mathfrak{n}_{i}=\mathfrak{n}_{j}^{\sigma}$ for some $\sigma \in G_{1}$. As $G_{1}$ acts trivially on $S / \mathrm{m} S$, there is a unique maximal ideal of $S$ over each maximal ideal of $S^{G_{1}}$. If $G_{1}$ has order $q=p^{a}$, then for any $f \in K_{i}, f^{q} \in S^{G_{1}} / \mathfrak{n}_{i}^{G_{1}}$, so that $S^{G_{1}} / \mathrm{n}_{i}^{G_{1}}=$ $K_{i}$ by separability. Thus the canonical map $S^{G_{1}} / \mathrm{m} S^{G_{1}} \rightarrow S / \mathrm{m} S$ is surjective. By Nakayama's lemma $S^{G_{1}}=S$ and $G_{1}=(1)$. Therefore $G$ acts faithfully on $S / \mathrm{m} S$.

Let $K$ be the fraction field of $S$. Then $K^{G}$ is the fraction field of $R$ and $K=K^{G} \otimes_{R} S_{1}$ which shows that $S$ is free of rank $g=$ order of $G$ over $R$. Hence $[S / \mathrm{m} S: k]=g$. Since $G$ is transitive on the $K_{i}, K_{0}=(S / \mathrm{m} S)^{G}$ is an extension field of $k$. If this is the stabilizer of $K_{i}$ in $G$, then $K_{0}$ maps onto $K_{i}^{H_{i}}$. Let $h$ be the common order of the conjugate subgroups $H_{i}$ of $G$. Then $\left[S / \mathrm{m} S: K_{0}\right]=r h=g$, so $K_{0}=k$. Choose $\delta \in K$, so that $\left\{\delta^{\sigma}: \sigma \in H_{1}\right\}$ is a basis of $K_{1}$ over $k$. Let $f \in S$ map to $\delta \bmod m S$, Then $\left\{\delta^{\sigma}: \sigma \in G\right\}$ is a $k$-basis of $S / \mathfrak{m} S$ and so, by Nakayama's lemma, $\left\{f^{\sigma}: \sigma \in G\right\}$ is a free basis of $S$ over $R$.

Now let $R$ be a noetherian local ring with maximal ideal $m$. Let $X=\operatorname{Spec}(R)$ and let $\dot{X}=X-\{\mathfrak{m}\}$ be the punctured spectrum of $R$. Recall that $\operatorname{depth}(R)=$ $\left\{q: H_{\mathrm{m}}^{q}(R) \neq(0)\right\}$, where $H_{\mathrm{m}}^{\cdot}$ denotes the local cohomology (cf. [3]). One also has 
an exact sequence

$$
0 \rightarrow H^{0}(R) \rightarrow R \rightarrow H^{0}\left(\dot{X}, \theta_{X}\right) \rightarrow H^{1}(R) \rightarrow 0
$$

and isomorphisms $H^{q}\left(\dot{X}, \Theta_{X}\right) \stackrel{\sim}{\rightarrow} H_{\mathfrak{m}}^{q+1}(R)$ for $q>0$. Thus $\operatorname{depth}(R)<\sup (2, q)$ whenever $H^{q-1}\left(\dot{X}, \Theta_{X}\right) \neq(0)$.

If $Y$ is an affine scheme and $G$ is a finite group acting faithfully on $Y$, we say that $G$ acts freely on $Y$ if the map $Y \rightarrow \operatorname{Spec}\left(H^{0}\left(Y, \theta_{Y}\right)^{G}\right)$ is étale. For an arbitrary scheme $Y$ we say that $G$ acts freely on $Y$ if there is a cover of $Y$ by $G$-stable affine opens $Y_{i}$ such that $G$ acts freely on each $Y_{i}$.

Proposition 4. Let $R$ be a noetherian local domain with maximal ideal $m$ and let $X=\operatorname{Spec}(R)$. Let $p>0$ be the characteristic of $R / m$ and let $G$ be a cyclic group of $\operatorname{order} p^{n} h, n>0$, acting on $R$ such that

(i) $R^{G}$ is noetherian and $R$ is a finite $R^{G}$-module;

(ii) $G$ operates trivially on $R / \mathrm{m}$;

(iii) $G$ acts freely on $\dot{X}$.

\section{Then}

(a) if $R$ is equicharacteristic of depth $>2$, then $\operatorname{depth}\left(R^{G}\right)<2$;

(b) if $\operatorname{depth}(R) \geqslant 3$, then $\operatorname{depth}\left(R^{G}\right)<3$.

ReMarK. The hypothesis (i) will be satisfied if the module $\Omega_{(R / p R) / z}$ of Kähler differentials of $R / p R$ is a finite $R$-module (see [1]).

To prove the proposition, we first show that, in case (a), $H^{1}(G, R) \neq(0)$, and in case (b), $H^{2}(G, R) \neq(0)$. In either case if $\sigma$ is a generator of $G$, we have

$$
\begin{aligned}
& H^{1}(G, R)=(\text { elements of trace } 0) /\left(\text { all } f^{\circ}-f, f \in R\right), \\
& H^{2}(G, R)=R^{G} / \text { (traces). }
\end{aligned}
$$

Since $G$ operates trivially on $R / \mathfrak{m}$, we have $f^{\sigma}-f \in \mathfrak{m}$ for all $f \in R$, and so $1 \neq f^{o}-f$ for any $f$. But in case $(a), \operatorname{trace}(1)=0$. In addition, 1 is not a trace, for if $1=\operatorname{trace}(f)$, then in $R / \mathfrak{m}$ we have $1=p^{n} h \bar{f}=0$, where $\bar{f}$ is the residue of $f$.

Let $Y=\operatorname{Spec}\left(R^{G}\right)$. Then $\dot{Y}$ is a geometric quotient of $\dot{X}$ by the action of $G$ (see [5]). There are two spectral sequences with the same abutment, whose $E_{2}^{p, q}$ terms are

$$
\begin{array}{ll}
\text { I: } \quad & H^{q}\left(G, H^{p}\left(\dot{X}, \vartheta_{X}\right)\right), \\
\text { II: } \quad H^{p}\left(\dot{Y}, \underline{H}^{q}\left(G, \vartheta_{X}\right)\right),
\end{array}
$$

where $\underline{H}^{q}\left(G, \theta_{X}\right)$ is the sheaf associated to the presheaf on $\dot{Y}$, थ $\mapsto H^{q}\left(G, H^{0}\left(V, \theta_{X}\right)\right), V$ being the inverse image of $\mathcal{U}$. By Lemma 3, $\underline{H}^{q}\left(G, \theta_{X}\right)=(0), q>0$, and II collapses. Thus we have a single spectral sequence $H^{q}\left(G, H^{p}\left(\dot{X}, \vartheta_{X}\right)\right) \Rightarrow H^{*}\left(\dot{Y}, \vartheta_{Y}\right)$. Since $\operatorname{depth}(R)>2$, it follows that $H^{0}\left(\dot{X}, \vartheta_{X}\right)=$ $R$ and the exact sequence of terms of low degree is

$$
0 \rightarrow H^{1}(G, R) \rightarrow H^{1}\left(\dot{Y}, \theta_{Y}\right) \rightarrow H^{1}\left(\dot{X}, \theta_{X}\right)^{G} \rightarrow H^{2}(G, R) \rightarrow H^{2}\left(\dot{Y}, \theta_{Y}\right) \text {. }
$$

In case (a), $H^{1}(G, R) \neq(0)$, so $H^{1}\left(\dot{Y}, \theta_{Y}\right) \neq(0)$, and $\operatorname{depth}\left(R^{G}\right)>2$. In case (b), $H^{1}\left(\dot{X}, \vartheta_{X}\right)=(0)$ since $\operatorname{depth}(R)>3$. But $H^{2}(G, R) \neq(0)$, so $H^{2}\left(\dot{Y}, \theta_{Y}\right) \neq(0)$ and $\operatorname{depth}\left(R^{G}\right)<3$. Q.E.D. 
Combining case (a) with Proposition 2 shows that one always has $\operatorname{depth}\left(R^{G}\right)=$ 2 , provided depth $(R) \geqslant 2$.

3. An example. We give an example which shows that the inequality of case (b) in Proposition 4 cannot be improved. Let $A$ be the ring of Gaussian integers and let $R_{0}$ be the localization of $A$ at the prime $(1+i) A$. Complex conjugation induces an automorphism of $R_{0}$. The residue field of $R_{0}$ is $F_{2}$. Let $S=$ $R_{0}\left[X_{1}, \ldots, X_{n}, Y_{1}, \ldots, Y_{n}\right]$ be the ring of polynomials over $R_{0}$ in the two sets of variables $\underline{X}=\left(X_{1}, \ldots, X_{n}\right), \underline{Y}=\left(Y_{1}, \ldots, Y_{n}\right)$ and let the group $G$ of order 2 operate on $S$ as follows. If $f \in S$, write $f=\Sigma a_{\alpha \beta} \underline{X}^{\alpha} \underline{Y}^{\beta}$-in the usual notation, $\alpha=\left(\alpha_{1}, \ldots, \alpha_{n}\right)$, etc. Let $\bar{f}=\sum \bar{a}_{\alpha \beta} \underline{Y}^{\alpha} \underline{X}^{\beta}$. Let $V=\operatorname{Spec}(S), V_{0}=\operatorname{Spec}\left(R_{0}\right)$. If $x$ is the closed point and $\zeta$ the generic point of $V_{0}$, then $V_{x}=\operatorname{Spec}\left(F_{2}[\underline{X}, \underline{Y}]\right)$, and $V_{\xi}=\operatorname{Spec}(Q(i)[\underline{X}, \underline{Y}])$. Also $V^{G} \subset V_{x}^{G}$, for if $y$ is a point of $V-V_{x}$, then $\mathbf{Q}(i)$ is contained in the residue field $\underline{\kappa}(y)$ at $y$, so if $G$ leaves $y$ fixed, it operates nontrivially in $\kappa(y)$. Thus $V^{G}$ is defined by the ideal

$$
I=\left(2, X_{1}-Y_{1}, \ldots, X_{n}-Y_{n}\right) \text {, }
$$

in $S$. Let $P=\sqrt{I}$. Then $P$ is prime in $S$. Let $R=S_{p}$. Then the residue field of $R$ is the function field of $V^{G}$, upon which $G$ operates trivially.

Let $\bar{S}=S /(1+i) S \approx F_{2}[\underline{X}, \underline{Y}]$. Every invariant in $\bar{S}$ is a sum of elements of the form

$$
\underline{X}^{\alpha} \underline{Y}^{\alpha} \underline{Y}^{\alpha}+\underline{X}^{\beta} \underline{Y}^{\alpha}, \quad \alpha \neq \beta,
$$

On the other hand, every invariant in $S$ is a sum of elements of the form

$$
\begin{gathered}
a \underline{X}^{\alpha} \underline{Y}^{\beta}+\bar{a} \underline{X}^{\beta} \underline{Y}^{\alpha}, \quad \alpha \neq \beta, a \in R_{0}, \\
b \underline{X}^{\alpha} \underline{Y}^{\alpha}, \quad b \in R_{0} \cap \mathbf{Q} .
\end{gathered}
$$

Thus $\bar{S}^{G}=S^{G} /((1+i) S)^{G}$, and so if $R$ denotes $\bar{R} /(1+i) R$, then $\bar{R}^{G}=$ $R^{G} /((1+i) R)^{G}$.

We show now that 2 is a prime element of $R^{G}$. For this it suffices to show that 2 is prime in $S^{G}$, i.e., if $f \in S$ is such that $(1+i) f \in S^{G}$, then $(1+i) f=2 g$, $g \in S^{G}$. (Since $(1+i)$ is prime in $R_{0}$, it is prime in $S$.) However $(1+i) f \in S^{G}$ implies $\bar{f}=i f$, i.e., the coefficients $a_{\alpha \beta}$ of $f$ satisfy $\bar{a}_{\alpha \beta}=i a_{\beta \alpha}$ and we get $i\left(a_{\alpha \beta} \pm a_{\beta \alpha}\right)=\overline{a_{\alpha \beta} \pm a_{\beta \alpha}}, \alpha \neq \beta$, and $i a_{\alpha \alpha}=\overline{a_{\alpha \alpha}}$. Thus $a_{\alpha \beta}=c_{\alpha \beta}(1-i), c_{\alpha \beta} \in R_{0} \cap$ Q. Also

$$
c_{\alpha \beta}(1+i)=i c_{\alpha \beta}(1-i)=\overline{c_{\beta \alpha}(1-i)}=c_{\beta \alpha}(1+i),
$$

so $c_{\alpha \beta}=c_{\beta \alpha}$. Let $g=\sum c_{\alpha \beta} \underline{X}^{\alpha} \underline{Y}^{\beta}$. Then $g \in S^{G}$ and $f=(1-i) g$.

Therefore $R^{G} / 2 R^{G}=\bar{R}^{G}$. $R$ (resp., $\bar{R}$ ) is a regular local ring of dimension $n+1$ (resp., $n$ ). If the hypotheses of Proposition 4 are satisfied for $R$, they are also satisfied for $\bar{R}$, and to prove them for $R$, it suffices to show that the quotient map $\phi: V \rightarrow V / G=\operatorname{Spec}\left(R^{G}\right)$ is étale on $V-V^{G}$. If $K$ is the fraction field of $S$, then $K^{G}$ is the fraction field of $S^{G}$ and $K^{G} \otimes_{S^{G}} S=K$. Also, for all closed points $z \in \phi\left(V-V^{G}\right)$ we have

$$
\operatorname{dim}_{\kappa(z)} S \otimes_{S^{G}} \kappa(z)=\left[K: K^{G}\right]=2,
$$


from which it follows that $\phi \mid V-V^{G}$ is flat. Since the residue fields of closed points of $V / G$ are either finite or of characteristic 0 , it follows that all such residue field extensions are separable. Finally, $\phi\left(V-V^{G}\right)$ is exactly the set over which $\phi$ is unramified. Therefore, taking $n \geqslant 2$ and applying case (a) to $\bar{R}$, we get $\operatorname{depth}\left(R^{G}\right)$ $=3$.

\section{REFERENCES}

1. J. Fogarty, Kahler differentials and Hilbert's fourteenth problem for finite groups, Amer. J. Math. 102 (1980), 1159-1175.

2. A. Grothendieck, Sur quelques points d'algèbre homologique, Tôhoku Math. 9 (1957), 119-221.

3. __ Local cohomology, Lecture Notes in Math., vol. 41, Springer-Verlag, Berlin and New York, 1967.

4. J. Lipman, Unique factorization in complete local rings, Proc. Sympos. Pure Math., vol. 29, Amer. Math. Soc., Providence, R.I., 1975, pp. 531-546.

Department of Mathematics, University of Massachusetts, AmHerst, Massachusetts 01003 